\title{
Study of spin waves in magnetic thin films submitted to external mechanical stresses.
}

\author{
W. Karboul ${ }^{1}$, D. Faurie ${ }^{1, a}$, S. M. Chérif ${ }^{1}$, P. Djemia ${ }^{1}$, P.-O. Renault ${ }^{2}$, Ph. Goudeau ${ }^{2}$ \\ ${ }^{1}$ LPMTM, Université Paris13, 93430 Villetaneuse, France \\ ${ }^{2}$ LPM PhyMat, Université de Poitiers, 86962 Futuroscope, France
}

Magnetic thin layers show properties that may differ from their bulk counterparts. Understanding the properties of these systems requires an accurate characterisation of their structure and their morphology, in order to determine the influence of mechanical stresses and reduced sizes. In order to accurately study the elastic properties of thin films, we have developed mechanical testing in-situ coupled with x-ray diffraction [1]. This method is not yet widely used to study such systems. The principle is to apply known stresses to thin films, while intra-granular strains are determined by $\mathrm{x}$-ray diffraction.

In the case of magnetostrictive materials, such as $\mathrm{Ni}$, when the film is elastically strained, its magnetic anisotropy can change. This effect is called inverse magnetostriction. In the most of cases, thin magnetic layers are strained during their fabrication (due to thermic and epitaxial stresses). These strains are directly related to structural parameters (crystallographic texture, thickness) that can play a role too, it is thus difficult to know with accuracy the only strain contribution to the magnetic anisotropy in thin films. We develop at LPMTM an original technique that combines in-situ tensile tests on ferromagnetic films (deposited on soft polyimide substrate) under external applied magnetic field with the Brillouin light scattering (BLS) technique [2]. We are able to vary the applied mechanical stresses intensity in a controllable manner in the films.

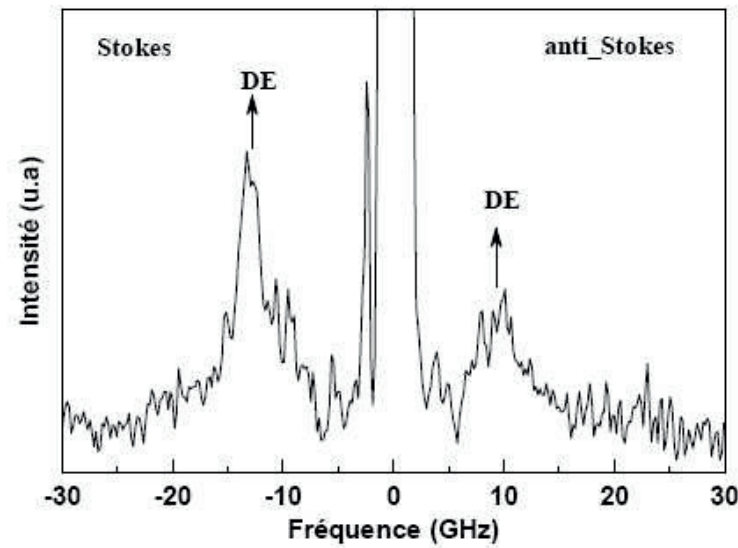

Figure 1: Magnetic BLS spectrum from a Ni film $(200 \mathrm{~nm})$ on polyimide substrate. DE denotes the Damon Eschbach surface spinwave (external magnetic field $\mathrm{H}=500 \mathrm{Oe}$ and the angle of incidence of light is $45^{\circ}$ ).

a e-mail : faurie@1pmtm.univ-paris13.fr 
We analyzed the frequency position of spin waves observed by BLS in order to deduce the magnetic characteristic of the studied Nickel layers (Figure 1). Their variations under controlled external stress modification enable us to measure some magneto-elastic coupling constants or magnetostrictive coefficients.

In the same time, we can also observe acoustic waves (see figure 2) from which we can deduce the average effective elastic constants of the whole material.

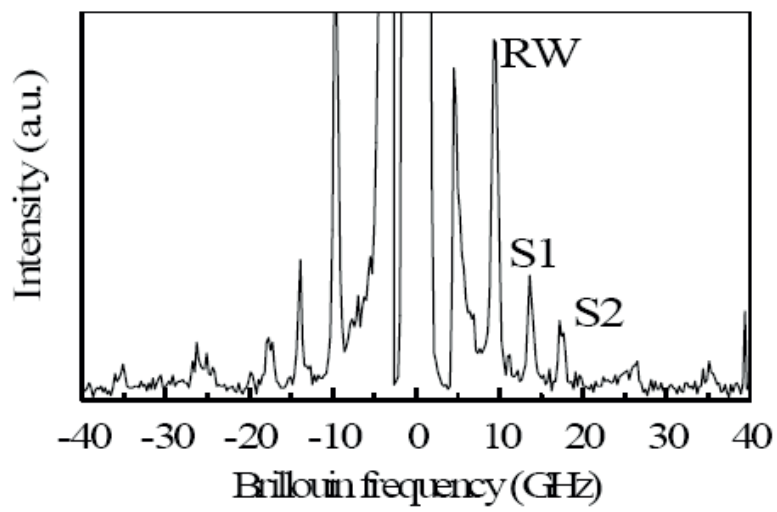

Figure 2: Acoustic BLS spectrum from a Ni film (200 nm) on polyimide substrate. RW denotes the Rayleigh surface wave, Si the ith Sesawa wave (the angle of incidence of light is $45^{\circ}$ ).

These average elastic constants will be confronted to elastic constants of each phase founded by $\mathrm{x}$-ray diffraction, after an appropriate homogenization procedure. The knowledge of these elastic properties is necessary for magnetic anisotropy calculation that comes from magnetoelastic coupling.

[1] D. Faurie, P. -O. Renault, E. Le Bourhis, P. Goudeau, Acta Materialia 54, 4503 (2006)

[2] M. Belmeguenai, F. Zighem, Y. Roussigné, S.M. Chérif, P.Moch, K. Westerholt, G. Woltersdorf, G. Bayreuther, Physical Review B 79, 024419 (2009) 